\title{
Wind erosion mechanism of cement-mortar protective layer under air-borne sand environment
}

\author{
Ying Jia ${ }^{1, \text { a }}$, Bei Lee ${ }^{2, b}$, Mei Zhang ${ }^{3, c}$,Shuaifeng Hao ${ }^{4, d}$ \\ ${ }^{1}$ School of Civil Engineering, Beijing Jiaotong University Beijing 100044,China \\ 2,3,4 School of Civil Engineering, Beijing Jiaotong University Beijing 100044,China

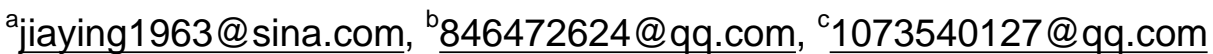

Keywords: Wind erosion; Wind erosion critical pressure; Elastic modulus attenuation; Sand impact Abstract. The paper simulated numerically the process of wind-blown sand flow impact-induced erosion of cement-mortar block. The concept of wind erosive critical pressure was put forward. Furthermore, the calculation formula of the impact pressure of sand grains, the attenuation equation of elastic modulus and the calculation formula of sand impact times occurred are deduced when the elastic modulus is known, and the minimum elastic modulus value of wind erosion is deduced.

\section{Introduction}

Wind erosion refers to the erosion to the construction and buildings by air-borne sand, namely, the physical process of solid surface material loss induced by the concentration flow constantly hitting solid surface ${ }^{[1]}$. For example, in the western of China, the strong wind, which is usual there, brings the air-borne sand erosion to the construction, particularly to the reinforced concrete bridges which are an important part of the traffic artery.

The elastic modulus is increasingly undermined with the growing of the initial crack at concrete surface of bridge under the effect of air-borne sand, finally caused the surface peeling and wind erosion appear. Wind erosion is one of the damages to bridges in the air-borne sand areas, it aggravates the concrete carbonizing, disoperation from chloride and freeze-thaw damage and causes the concrete structure deteriorate greatly. The research of the wind erosion along the surface of bridge under air-borne sand environment has great practical significance in the preserve of quality of existing bridges ${ }^{[2]}$.

\section{The Definition of Critical Pressure}

In 2013, Yanping Wang and et al studied the effect of motar blocks under different speed, impact angle, erosion time and the amount of sand flux by wind-blown sand flow jet method. It is concluded that the erosion happened when the sand flux speed reached $13.54 \mathrm{~m} / \mathrm{s}$ and impacted vertically for 3 minutes ${ }^{[3]}$. Accordingly this paper defines $13.54 \mathrm{~m} / \mathrm{s}$ as the critical speed of wind erosion, namely the minimum speed of wind-blown sand flow that can erode the protective layer mortar.

In this paper, the experimental parameters were used ${ }^{[3]}$ to do the numerical simulation and got pressure contour map at the erosion surface, as shown in Fig.1.

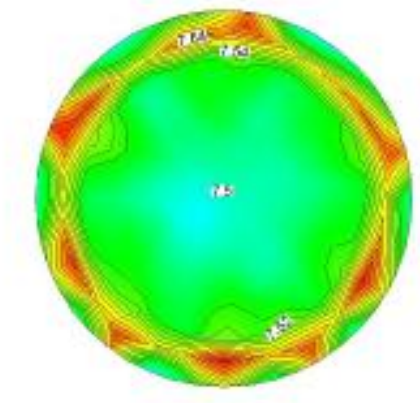

Fig.1 Pressure contour map in the erosion surface [unit: $\mathrm{kPa}$ ] 
The pressure at the erosion surface of cement-mortar block distributes evenly in the central region and increases gradually from the center to the edge. The numerical simulation use the critical speed of wind erosion, so the pressure at the erosion surface in Fig.2.1 can be regarded as the critical pressure of wind erosion on the surface of cement-mortar test block.

The critical pressure is defined as $7.5 \mathrm{kPa}$, and the minimum pressure of the cement-mortar protective layer appears wind erosion according to the Fig.2.1. In other words, the cement-mortar protective layer began to wind erosion when the pressure reached $7.5 \mathrm{kPa}$ and impacted the windward surface of bridge for 3 minutes.

\section{The Deduction of Calculation Formula of Sand Impact Pressure}

To derive the impact pressure of sand to cement mortar, sand can be simplified as a ball with radius $R$, mass $m$, elastic modulus $E_{1}$ and Poisson's Ratio; cement mortar can be simplified as semi-infinite space with elastic modulus $E_{2}$ and Poisson's Ratio. Sand are set to strike cement mortar at an incident angle $\theta$ with the velocity of $V_{0}$. Such as Fig.2

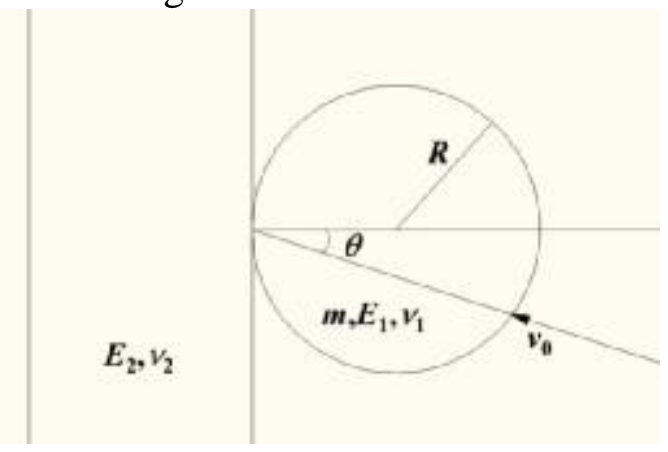

Fig.2 Physical model of impact of sand to cement mortar

The impact of sand to cement mortar can be regarded as an elastic impact before wind erosion (including the critical state of wind erosion) because cement mortar is quasi-brittle material. Because of the large elastic modulus of pellets and cement mortar, $a<<$.

Hertz gives the normal compressive stress as:

$$
p(r)=p_{0}\left(a^{2}-r^{2}\right)^{\frac{1}{2}} / a
$$

The radius of the contact circle is:

$$
a=\left(\frac{3 p_{\max } R}{4 E^{*}}\right)^{\frac{1}{3}}
$$

Distance between two objects close to each other:

$$
\delta=\pi a p_{0} / 2 E^{*}
$$

The total load on the cement mortar and the relationship between pressures:

$$
\begin{gathered}
P=\int_{0}^{a} p(r) 2 \pi r \mathrm{~d} r=\frac{2}{3} p_{0} \pi a^{2} \\
p_{\max }=\left(\frac{125}{64} m k^{2} V_{z 0}{ }^{6}\right)^{1 / 5}
\end{gathered}
$$

That is, the calculation formula of sand impact pressure is:

$$
F_{0}=\frac{2}{\pi}\left(\frac{5}{16} m\left(V_{0} \cos \theta\right)^{2} R^{-3} E^{* 4}\right)^{\frac{1}{5}}
$$

in which $\frac{1}{E^{*}}=\frac{1-v_{1}^{2}}{E_{1}}+\frac{1-v_{2}^{2}}{E_{2}}, k=\frac{4}{3} R^{1 / 2} E^{*}$, and $V_{Z 0}=V_{0} \cos \theta$ is the perpendicular component to cement mort ar of sand speed. 
When the elastic modulus of cement mortar $E_{2}$ is a variable and its variation range is $0 \sim 10 \mathrm{MPa}$, the other parameters are fixed values, then $E^{*} \approx E_{2}$. We can get the relationship between $F_{0}$ and $E_{2}$ from $\mathrm{Eq}$ (3.6) when set $E_{2}=E$, as shown in Fig.3

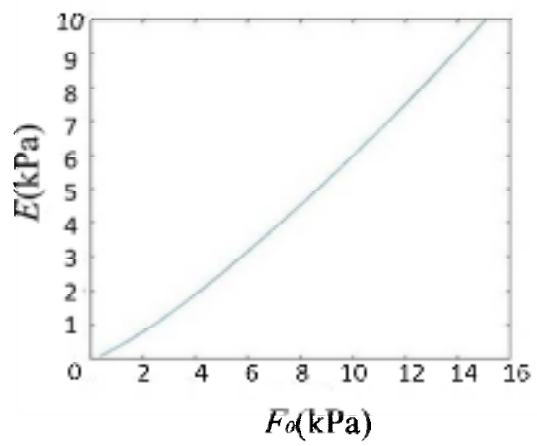

Fig.3 Function relationship between $F_{0}$ and elasticity modulus $E$

It can be seen from Fig. 3.2 that the pressure of the surface of sand mortar striking cement mortar increases with the increase of elastic modulus $E$.

\section{The Analysis of Wind-Erosion Mechanism}

The crack begins to propagate when the sand impacted the cement mortar layer first time, the elastic modulus $E$ of cement mortar is reducing with the crack propagation and the impact force $F_{0}$ next time between sand and cement mortar is reducing accordingly; also the half length of crack $a$ is growing and the impact pressure needed for next crack propagation was reducing too. Sequentially derivation, the impact force $F_{0}$ and the elastic modulus form a feedback mechanism, and until $E$ declined to $E^{0}$, the wind erosion happens. This process reflected the time effects of wind erosion, namely, the wind erosion is the result of repeated sand impact.

According to the feedback mechanism of $F_{0}$ and $E_{2}$, the elastic modulus $E^{*}$ is reducing with the growing number of sand impact, set the attenuation formula of the elastic modulus $E^{*}$ as:

$$
E_{n+1}^{*}=\frac{\kappa E_{n}^{*}}{\left(\frac{F_{0}}{7.5^{*} 10^{\wedge}-3}\right)^{\gamma}}
$$

where $\kappa$ and $\gamma$ are constants, $E_{\mathrm{n}}$ is the remaining elastic modulus of cement mortar when the sand impacts the cement mortar for the $n$th time and $F_{\mathrm{n}}$ is the impact force of sand to cement mortar when the remaining elastic modulus is $E_{\mathrm{n}}$.

Set:

$$
\beta=\frac{1}{\left(\frac{2}{\pi}\left(\frac{5}{16} \mathrm{mV}_{0}^{2} \mathrm{R}^{-3}\right)^{\frac{1}{5}} \times 7.5 \times 10^{3}\right)^{\gamma}}=\frac{1}{\left(4.3592 \times 10^{3}\right)^{\gamma}}
$$

Then the following can be derived:

$$
E_{n+1}^{*}=\frac{\kappa \beta E_{n}^{*}}{E_{n}^{* \frac{4}{5}} \gamma}=\kappa \beta E_{n}{ }^{*\left(1-\frac{4}{5} \gamma\right)} \quad(0<\gamma<1)
$$

Because the elastic modulus $E_{\mathrm{n}}$ finally converges to $E^{0}$ that is the elastic modulus when the wind erosion happens, namely, the minimum elastic modulus,

$$
\lim _{n \rightarrow \infty} \frac{E_{n+1}^{*}}{E_{n}^{*}}=\lim _{n \rightarrow \infty} \kappa \beta E_{n}^{*-\frac{4}{5} \gamma}=1
$$

According to the attenuation recursive formula (4.3) of $E^{*}$, then there is: 
Theoretically:

$$
E_{n}^{*}=(\kappa \beta)^{\frac{5}{4 \gamma}\left(1-\left(1-\frac{4 \gamma}{5}\right)^{n}\right)} E_{0}^{*\left(1-\frac{4}{5} \gamma\right)^{n}} \quad(n=1,2 \ldots)
$$

$$
\lim _{n \rightarrow \infty} E_{n}^{*}=\lim _{n \rightarrow \infty}(\kappa \beta)^{\frac{5}{4 \gamma}\left(1-\left(1-\frac{4 \gamma}{5}\right)^{n}\right)} E_{0}^{*\left(1-\frac{4}{5} \gamma\right)^{n}}=E^{0} \Rightarrow \kappa \beta^{\frac{5}{4 \gamma}}=E^{0}
$$

We can get the attenuation recursive formula of $E^{*}{ }_{n}$ :

$$
E_{n}^{*}=\left(E^{0}\right)^{\frac{4 \gamma}{5}} E_{n-1}^{*\left(1-\frac{4}{5} \gamma\right)}
$$

Then, the elastic modulus $E^{*}$ of attenuation equation can be written by:

$$
E_{n}^{*}=\left(E^{0}\right)^{\left(1-\left(1-\frac{4 \gamma}{5}\right)^{n}\right)} E_{0}^{*\left(1-\frac{4}{5} \gamma\right)^{n}} \quad(n=1,2 \ldots)
$$

where $E^{0}$ is the initial elastic modulus of cement mortar.

\section{Conclusions}

This paper defines the critical pressure of wind erosion of cement-mortar surface. It derived the formula of pressure caused by sand impact. According to the fracture mechanics, the minimum impact pressure is analyzed and derived when the crack of cement mortar develops, namely, the initial stress of crack propagation. With the analysis of wind erosion mechanism, the equation of elastic modulus attenuation was obtained, which illuminates the wind erosion process and the relationship between the elastic modulus of cement mortar and sand impact times. The numerical results in this paper provide a reference for studying the surface wind erosion of concrete bridges.

\section{Acknowledgements}

Acknowledgements the work is supported by National Natural Science Foundation of China [No. 51778051].

\section{References}

[1]LI Shuguang, CHEN Gaixin, JI Guojin, LU Yi-hui. Quantitative analysis of initial microcrack in high strength concrete by DIP technique. Concrete, 2013,(5):6-12.

[2]ZHANG Yan, WANG Qicai, ZHANG Fengqin, MA Huajun. Comparative Experimental Research on Concrete Surface Treatment Materials Resistance to Wind Erosion. China Academy of Railway Sciences,2012,33(2):43-47.

[3]WANG Yanping, JU Chunchang, WANG Qicai. Experimental Study on the Solid Particle Erosion of Concrete, Mortar and Cement Paste under Blown Sand Enviroment. China Railway Science. 2013,34(5):21-25. 